\title{
HYALURONIDASE IN OCÚLAR TISSUES* II. HYALURONIDASE IN THE TISSUES OF THE RABBIT'S EYE
}

BY

\author{
G. MAYER, I. C. MICHAELSON, AND N. HERZ \\ Haifa
}

HYALURONIC acid has been found in considerable quantities in the vitreous humour (Meyer and Palmer, 1934) and hyaluronosulphate in the cornea (Meyer and Chaffee, 1940). The presence of the enzyme hyaluronidase in the iris and ciliary body has been referred to by Meyer and Palmer (1936) and Meyer and others (1936).

In testing theories regarding the physiological importance of hyaluronidase in the eye, and its role in certain pathological conditions, such as keratitis with vascularization, a very sensitive test for detecting the presence of hyaluronidase is required. In this laboratory a sensitive bacteriological method has been developed which enables hyaluronidase in concentrations as low as 0.01 turbidity reducing units (T.R.U.) per $\mathrm{ml}$. to be detected (Mayer, 1955). This method is based on the observation that certain strains of capsulated streptococci lose their capsules in the presence of hyaluronidase (Hirst, 1941; McClean, 1941a,b).

A series of experiments using this method has been made to determine the presence of hyaluronidase in the tissues of the rabbit's eye.

\section{Materials and Methods}

The eye tissues of normal rabbits were removed aseptically and immediately processed by one of the following methods:

(1) Grinding with sand;

(2) Mashing with a small metal squeezer;

(3) Mashing in a glass homogenizer.

The third was found to be the most suitable method for our purpose since the tissues could be ground up until an amorphous foamy mass was obtained. The whole material thus extracted could be transferred to a test-tube containing a culture of capsulated streptococci.

\section{Experiments}

(1) Rabbit's Iris.- Nine irises weighing 0.2 gr. were frozen and mashed in a glass homogenizer, $0.4 \mathrm{ml}$. saline being added during the process. A fairly homogeneous suspension was obtained, and $0.2 \mathrm{ml}$. was pipetted into a test-tube containing capsulated streptococci. A series of known dilutions of commercial hyaluronidase was run simultaneously to produce a standard curve. All the tubes were incubated for $2 \mathrm{hrs}$ at $37^{\circ} \mathrm{C}$.;

*Received for publication June 14, 1955. 
smears of the bacterial suspensions were stained and the proportion of capsulated and decapsulated chains was noted (Fig. 1). Three control tubes were set up, saline being added instead of hyaluronidase.

(2) Traumatized Rabbit's Cornea.-Extensive thermal lesions were caused in the right corneae of two rabbits by means of an electro-cautery. On the following day the corneae were removed aseptically and mashed in a glass homogenizer; $0.2 \mathrm{ml}$. saline was added to the homogenizer and $0 \cdot 2 \mathrm{ml}$. of the resulting suspension was withdrawn with a pipette and added to the standard culture (Fig. 2).

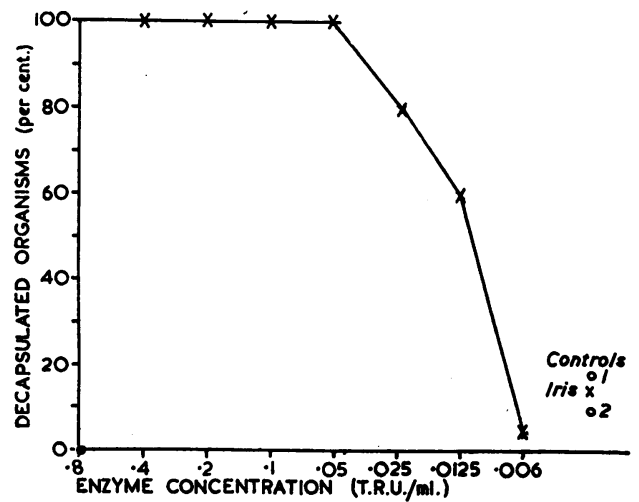

Fig. 1.-Estimation of hyaluronidase in normal rabbit's iris, with standard curve and two saline controls.

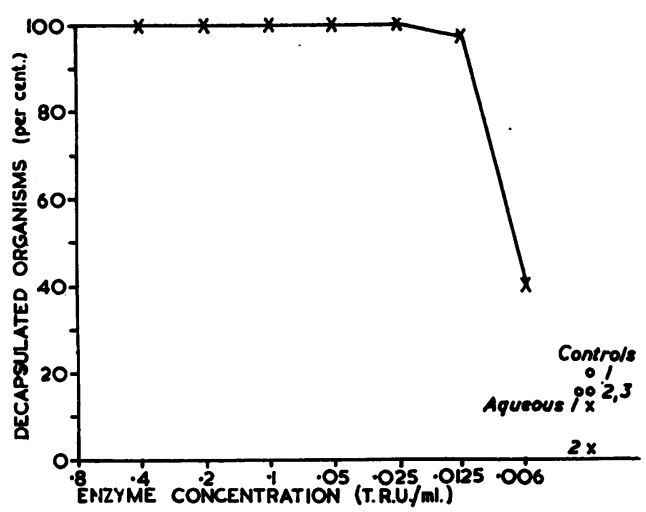

Fig. 3.-Estimation of hyaluronidase in aqueous humour of rabbit with standard curve and three saline controls.

uronidase corresponding to 0.025 T.R.U. caused decapsulation of at least 80 per cent. of the streptococci, whereas the control tubes contained 18 and 10 per cent. respectively of chains with very small capsules or without capsules. In the tube with the extracted irises there were 15 per cent. of streptococci with very small capsules or without capsules. This indicated that no hyaluronidase could be demonstrated in the pooled irises in a concentration as low as 0.025 T.R.U. per ml. 
Fig. 2 shows that a concentration of hyaluronidase corresponding to approximately 0.006 T.R.U. per ml. still caused decapsulation of 100 per cent. of the streptococci, whereas spontaneously decapsulated organisms amounted to only 5-14 per cent. of the control population. In the tube containing traumatized cornea there were 13 per cent. of streptococci with very small capsules, indicating that the traumatized corneae did not cause decapsulation of the streptococci. In the two corneae extracted no hyaluronidase could be detected in concentrations as low as 0.006 T.R.U. per $\mathrm{ml}$.

Fig. 3 shows that the series of dilutions of hyaluronidase caused decapsulation of nearly 100 per cent. of the streptococci down to a concentration of approximately 0.0125 T.R.U. per $\mathrm{ml}$. The concentration of 0.006 T.R.U. per ml. caused decapsulation in 38 per cent. of the streptococci. In the three controls, the percentage of chains without or with very small capsules was 20,15 , and 15 per cent. respectively. In the two tubes containing aqueous humour, the percentage of decapsulated streptococci was 13 and 2 per cent. respectively. Hyaluronidase was absent from the aqueous of the rabbit down to a concentration of 0.0125 T.R.U. per ml.

No hyaluronidase activity could be detected by these methods in the ciliary body, cornea, or autolysed iris of the rabbit.

\section{Discussion}

Our finding that no hyaluronidase activity can be demonstrated in the ocular tissues of the rabbit is at variance with other authors' positive findings. Two possible sources of error should be mentioned:

(a) The small quantities of hyaluronidase which might have been present in the tissue examined, might have become inactivated by our manipulation, and this might account for our negative findings.

(b) The hyaluronidase activity found by other authors might be due to extraneous factors, such as contamination by hyaluronidase-producing micro-organisms.

To exclude the former possibility the following recovery experiment was performed:

$0.25 \mathrm{ml}$. hyaluronidase containing a concentration of 0.5 T.R.U. per ml. was injected into the intact cornea of a rabbit. A bleb appeared and some of the injected fluid oozed out and was lost. The cornea was aseptically removed, washed, and ground in the glass homogenizer, and the contents were diluted and tested. The dilution of 1:10 of the homogenate gave evidence of hyaluronidase activity, proving that the enzyme had not become inactivated by manipulation.

With regard to the second possibility, it should be mentioned that on certain occasions, when we were using the plate method of Murray and Pearce (1949), hyaluronidase activity appeared to be encountered in the cornea of the rabbit.

In these instances we isolated a staphylococcus which had contaminated 
the cornea and found it to be hyaluronidase-producing. The hyaluronidase activity, which seemed to be due to the presence of the enzyme in the tissue, was thus in fact due to hyaluronidase elaborated by the contaminant.

\section{Summary}

The hyaluronidase contents of ocular tissues and fluids have been assayed on a capsulated streptococcus.

No hyaluronidase activity could be demonstrated in homogenates of the iris, ciliary body, normal cornea, wounded cornea, or aqueous fluid of the rabbit.

This work was carried out in the ophthalmic laboratory of the Rambam Government Hospital, Haifa, under the direction of Dr. I. C. Michaelson. It was supported by a grant from the Arnold Reuben Fight for Sight Fund of the National Council to Combat Blindness, Inc., New York.

\section{REFERENCES}

HiRst, G. K. (1941). J. exp. Med., 73, 493.

MCClean, D. (1941a). J. Path. Bact., 53, 13. (1941b). Ibid., 53, 156.

MAYER, G. (1955). British Journal of Ophthalmology, 39, 747.

MeYer, K., and Chaffee, E. (1940). Amer. J. Ophthal., 23, 1320.

, Dubos, R., and Smyth, E. M. (1936). Proc. Soc. exp. Biol., 34, 816 and Palmer, J. W. (1934). J. biol. Chem., 107, 629. (1936). Amer. J. Ophthal., 19, 859.

Murray, R. G. E., and Pearce, R. H. (1949). Canad. J. Res., Sect. E., 27, 254.

EDITORIAL NOTE: Independent confirmation of some of these findings has been recently published by Bárány, E., and Wood, A. M. (1955). Acta physiol. scand., 33. 258. 